\title{
Optimal personnel reallocation in production processes based on the skills and knowledge in the chemical industry
}

\author{
Hajime Eguchi ${ }^{\text {a, }}$, Tomomi Aoyama ${ }^{a}$, Kohei Seki ${ }^{a}$, Ichiro Koshijima ${ }^{a}$ \\ ${ }^{a}$ Nagoya Institute of Technology, Gokiso-cho, Showa-ku, Nagoya-city, Aichi-ken, 466-8555, Japan \\ * Corresponding author: h.e@ triton.ocn.ne.jp
}

\begin{abstract}
In the chemical industry, the level of skills and knowledge of production staff is important to maintain the productivity of production processes. In spite of the difficulty to measure the level of skills, its quantitative estimation is possible. Therefore, by using a metric to estimate the value of skills in the production process, the optimal personnel allocation of production processes enables to produce the margin of work hours in the production staff's daily routine. This margin of workload is utilized to improve the skills and knowledge of production staff, in order to advance the operational resilience (i.e. the capability to restrain the disruption occurred inside and outside a production process). Hence, in this paper, the most effective personnel reallocation strategy is examined. At first, based on the real measured work hours devoted to skills of production staffs, the value of skills for the production staff belonging to the production group is calculated. Subsequently, the personnel allocation strategy that realizes the minimal work hours devoted to skills, without the decrease of the total value of skills in the groups, is searched. The example in this paper is composed of ten production staffs and three groups. The final result shows the efficiency of this procedure.
\end{abstract}

Keywords: Chemical industry, Production process, Skills and knowledge, Personnel reallocation, Operational resilience

\section{Introduction}

In the chemical industry, the group of production staff executes the daily routine in the production process. As the level of skills and knowledge of each production staff is different, it is difficult to judge if the personnel allocation is appropriate to the requirement of a production process or not.
The optimal personnel assignment in a group of production process has already been proposed ${ }^{(1,2)}$, but its criterion is based on the skill level of the production staff, making it hard to reflect the property of a production process. However, it is possible to measure the work hours devoted to skills (Hereafter, referred to as WHDS); and the criterion to estimate the skills quantitatively is named as the "value of skills" (The value of skills is defined later) ${ }^{(3)}$. Therefore, the skills of production staff are available for the index of the production process property. And as to the production staffs in the same group it is possible to assign each job that that minimizes the total WHDS without a decrease of total value of skills ${ }^{(4)}$. Hence, by using the procedure proposed in this paper, it is possible to enhance the operational resilience (i.e. the capability to restrain the disruptive signals that are against the production process.) of the production process. Namely, this is achieved by the improvement of skills and knowledge of the production staff through their reallocation, which permits the reduction of WHDS without a decrease of the total values of skill. The reduction of WHDS produces the margin of the workload, and it is available for the improvement of skills and knowledge. The production staffs execute their daily routine using three types of skills:

(a) Operational skills that mainly require the use of hands and legs

(b) Memory skills that are required to execute a smooth operation

(c) Communication skills that are required to attempt mutual communication

In this paper, the reduction of WHDS without a decrease of the value of skills, achieved by the optimal reallocation of production staffs, is introduced by means of an example. 


\section{Optimal reallocation of production staffs between production processes}

The chemical industry is composed of several production processes, and the reallocation of production staff in the same group or between several groups is practiced in order to improve the productivity. And, it is possible to decide the optimal personnel allocation based on WHDS without a decrease of the current value of skills achieved by the production staff.

\subsection{Prerequisite conditions for personnel reallocation}

Assuming that $\mathrm{P} 1$ and $\mathrm{P} 2$ are two production staffs subject to personnel reallocation, the prerequisite conditions of personnel reallocation are as follows:

(a) The value of skills that P1 (or P2) executes in the current daily routine does not decrease if replaced with P2 (or P1).

(b) In the case that P1 is required to execute higher value of skills than the former value of skills at the former position, additional WHDS are permitted.

Here, the current value of skills of $\mathrm{P} 1$ and $\mathrm{P} 2$ are respectively $Q 1$ and $Q 2$. In the case of $Q 1 \geq Q 2$, if $\mathrm{P} 1$ will practice the value of skills $Q 2$ as a guaranteed level after the alternation, P1 will be performing its duty. However the value of skills already gained by $\mathrm{P} 1$ at the former position will decrease of $(Q 1-Q 2)$. Therefore, it is necessary for $\mathrm{P} 1$ to execute the value of skills $Q 1$ to increase the former value of skills achieved by $\mathrm{P} 2$. In this case, WHDS will increase, but P1 has to increase the former value of skills $Q 2$ to $Q 1$ as a new guaranteed level. Simultaneously, P2 at the new position has to increase the value of skills from its guaranteed level $Q 2$ to $Q 1$, which was being achieved by $\mathrm{P} 1$. If $\mathrm{P} 1$ and $\mathrm{P} 2$ can attain the new value of skills $Q 1$ as their new guaranteed levels, they both arrive at the new balance of the value of skills. In the case of $Q 1<Q 2$, the same procedure will be adapted. Using this criterion, they aim to maintain and improve each value of skills. After the alternation of P1 and P2, the new value of skills becomes the goal to be achieved by their efforts.

\subsection{Selection of production staffs to be changed}

As to the personnel reallocation, there are two ways to decide a pair.

(a) P1 and P2 are fixed in advance, so there is no flexibility about a pair.

(b) After the selection of ' $n$ ' candidates, ' $n$ ' pairs that give the maximum total value of skills after the reallocation are searched.

In the case of ' $n$ ' candidates, the value of skills expected after the personnel reallocation is calculated as described in Section 2.1: within ' $n$ !' cases (the permutation $n(=n !)$ ), ' $n$ ' pairs of production staffs that give the maximum total value of skills are the solution.

\section{Example of optimal personnel reallocation}

In this section, three groups are under examination, where the $1^{\text {st }}$ group is composed of three production staffs. This group is the same as the one utilized in our previous study regarding the optimal job assignment of production staffs ${ }^{(4)}$. The remaining two groups, where the numbers of staffs are four and three respectively, are added. Therefore, the total number of groups is three and the total number of production staffs is ten.

Table1. Current work hours devoted to skills $B$ and value of skills $Q$ of three groups

\begin{tabular}{|c|c|c|c|c|c|c|c|c|}
\hline \multicolumn{4}{|c|}{ Before Personal change } & Operation & Memory & \multirow{2}{*}{$b_{3}^{\mathrm{i}}$} & \multicolumn{2}{|c|}{ Total of $\mathrm{F}$ Value of skills } \\
\hline G. no. & ID no. & i & $v$ & $b^{\mathrm{i}}{ }_{1}$ & $b^{\mathrm{i}}{ }_{2}$ & & $b_{\text {total }}^{\mathrm{i}}$ & $Q^{\mathrm{i}}$ \\
\hline \multirow{5}{*}{1} & 1 & 1 & 1.2 & 91 & 41 & 26 & 158 & 166,34 \\
\hline & 11 & 2 & 1.0 & 118 & 47 & 10 & 175 & 151,43 \\
\hline & 101 & 3 & 0.8 & 95 & 40 & 5 & 140 & 112,00 \\
\hline & \multicolumn{3}{|c|}{$b_{\mathrm{k} \text { total } ;(\mathrm{k}=1,2,3)}^{\mathrm{i}}$} & 304 & 128 & 41 & & \\
\hline & \multicolumn{3}{|c|}{$b_{\text {total }}, Q_{\text {total }}$} & & & & 473 & 429,77 \\
\hline \multirow{6}{*}{2} & 2 & 1 & 1.2 & 98 & 45 & 30 & 173 & 184,39 \\
\hline & 12 & 2 & 1.0 & 115 & 50 & 10 & 175 & 151,74 \\
\hline & 102 & 3 & 0.8 & 100 & 50 & 15 & 165 & 132,00 \\
\hline & 103 & 4 & 0.8 & 90 & 50 & 5 & 145 & 116,00 \\
\hline & \multicolumn{3}{|c|}{$b_{\mathrm{k} \text { total } ;(\mathrm{k}=1,2,3)}^{\mathrm{i}}$} & 403 & 195 & 60 & & \\
\hline & \multicolumn{3}{|c|}{$b_{\text {total }}, Q_{\text {total }}$} & & & & 658 & 584,13 \\
\hline \multirow{6}{*}{3} & 3 & 1 & 1.2 & 100 & 50 & 25 & 175 & 181,02 \\
\hline & 13 & 2 & 1.0 & 110 & 45 & 25 & 180 & 163,62 \\
\hline & 104 & 3 & 0.8 & 80 & 60 & 10 & 150 & 120,00 \\
\hline & \multicolumn{3}{|c|}{$b_{\mathrm{k} \text { total } ;(\mathrm{k}=1,2,3)}^{\mathrm{i}}$} & 290 & 155 & 60 & & \\
\hline & \multicolumn{3}{|c|}{$b_{\text {total }}, Q_{\text {total }}$} & & & & 505 & 464,64 \\
\hline & \multicolumn{6}{|c|}{ Total WHDS, total value of skills } & 1636 & 1478,54 \\
\hline
\end{tabular}

Candidates of allocation change

\subsection{Optimization of personnel reallocation}

In Table 1, four production staffs are selected as the candidates of the reallocation (ID no. 101,102,103,104). All of these candidates are new-comers (ID no. 1, 2, 3: supervisor, ID no. 11, 12, 13: Deputy-supervisor), and they can be allocated in any group. The procedure to find four pairs of production staff is as follows.

(a) Search four pairs that give the maximum value of skills expected after the personnel reallocation.

(b) The procedure of the personnel reallocation of P1 and P2 is as follows. 
The value of skills is calculated by Eq. (1) ${ }^{(5)}$.

Value of skills $\boldsymbol{Q}$

$=$ WHDS $\boldsymbol{B} \times$ Skill's suitability relative to the position of staff member $\boldsymbol{W}$

$\times$ Level of skills and knowledge $\boldsymbol{V}$

The components of vector $\boldsymbol{W}$ and $\boldsymbol{V}$ are described in Appendix.

The value of skills before the personnel reallocation is $Q_{\text {lold }}$ for P1, $Q_{2 \text { old }}$ for $\mathrm{P} 2$, and that after the reallocation is $Q_{1 \text { new }}$ for $\mathrm{P} 2, Q_{2 \text { new }}$ for P1. In the following equations, which represent the swapping between $\mathrm{P} 1$ and $\mathrm{P} 2$, the subscripts of $b_{\mathrm{ij}}$ are $\mathrm{i}=1$ for WHDS of P1 before reallocation, and $i=2$ for WHDS of P2 before reallocation; while $\mathrm{j}=1,2,3$ stands for the classification of skills (i.e. operational, memory and communication, as described in Section 1). The subscript of $w_{\mathrm{j}}$ is $\mathrm{j}=1,2,3$ and corresponds to the classification of skills; while for $v_{\mathrm{i}}, \mathrm{i}=1$ stands for $\mathrm{P} 1$ and $\mathrm{i}=2$ for $\mathrm{P} 2$.

(a) If $Q_{\text {lold }} \geq Q_{2 \text { old }}$, then $b_{\text {ijnew }}$ is decided as follows.

$$
\begin{aligned}
Q_{1 \text { new }}= & Q_{1 \text { old }} \\
Q_{2 \text { new }}= & Q_{1 \text { old }} \\
Q_{1 \text { new }}= & v_{1} \cdot\left(b_{11} \cdot w_{1}+b_{12} \cdot w_{2}+b_{13} \cdot w_{3}\right) \\
= & v_{2} \cdot\left(\left(v_{1} / v_{2}\right) \cdot b_{11} \cdot w_{1}\right. \\
& \left.+\left(v_{1} / v_{2}\right) \cdot b_{12} \cdot w_{2}+\left(v_{1} / v_{2}\right) \cdot b_{13} \cdot w_{3}\right) \\
b_{11 \text { new }}= & \left(v_{1} / v_{2}\right) \cdot b_{11} \\
b_{12 \text { new }}= & \left(v_{1} / v_{2}\right) \cdot b_{12} \\
b_{13 \text { new }}= & \left(v_{1} / v_{2}\right) \cdot b_{13} \\
Q_{2 \text { new }}= & v_{1} \cdot\left(b_{11} \cdot w_{1}+b_{12} \cdot w_{2}+b_{13} \cdot w_{3}\right) \\
= & v_{2} \cdot\left(b_{21} \cdot w_{1}+b_{22} \cdot w_{2}+b_{23} \cdot w_{3}\right) \cdot\left(Q_{1 \text { old }} / Q_{2 \text { old }}\right) \\
= & v_{1} \cdot\left(\left(v_{2} / v_{1}\right) \cdot b_{21} \cdot w_{1}+\left(v_{2} / v_{1}\right) \cdot b_{22} \cdot w_{2}\right. \\
& \left.+\left(v_{2} / v_{1}\right) \cdot b_{23} \cdot w_{3}\right) \cdot\left(Q_{1 \text { old }} / Q_{2 \text { old }}\right)
\end{aligned}
$$

$b_{21 \text { new }}=\left(v_{2} / v_{1}\right) \cdot b_{21} \cdot\left(Q_{1 \text { old }} / Q_{2 \text { old }}\right)$

$b_{2 \text { new }}=\left(v_{2} / v_{1}\right) \cdot b_{22} \cdot\left(Q_{1 \text { old }} / Q_{2 \text { old }}\right)$

$b_{23 \text { new }}=\left(v_{2} / v_{1}\right) \cdot b_{23} \cdot\left(Q_{\text {lold }} / Q_{2 \text { old }}\right)$

(b) If $Q_{\text {lold }}<Q_{2 \text { old }}$, then $b_{\text {ijnew }}$ is decided as follows.

$$
\begin{aligned}
Q_{\text {1new }} & =Q_{2 \text { old }} \\
Q_{2 \text { new }} & =Q_{2 \text { old }} \\
Q_{\text {1new }} & =v_{2} \cdot\left(b_{21} \cdot w_{1}+b_{22} \cdot w_{2}+b_{23} \cdot w_{3}\right) \\
= & v_{2} \cdot\left(\left(v_{1} / v_{2}\right) \cdot b_{11} \cdot w_{1}+\left(v_{1} / v_{2}\right) \cdot b_{22} \cdot w_{2}\right. \\
& \left.+\left(v_{1} / v_{2}\right) \cdot b_{23} \cdot w_{3}\right) \cdot\left(Q_{2 \text { old }} / Q_{1 \text { old }}\right) \\
b_{21 \text { new }} & =\left(v_{1} / v_{2}\right) \cdot b_{11} \cdot\left(Q_{2 \text { old }} / Q_{1 \text { old }}\right) \\
b_{2 \text { new }}= & \left(v_{1} / v_{2}\right) \cdot b_{12} \cdot\left(Q_{2 \text { old }} / Q_{1 \text { old }}\right) \\
b_{23 \text { new }}= & \left(v_{1} / v_{2}\right) \cdot b_{13} \cdot\left(Q_{2 \text { old }} / Q_{1 \text { old }}\right) \\
Q_{2 \text { new }}= & v_{2} \cdot\left(b_{21} \cdot w_{1}+b_{22} \cdot w_{2}+b_{23} \cdot w_{3}\right) \\
= & v_{1} \cdot\left(\left(v_{2} / v_{1}\right) \cdot b_{21} \cdot w_{1}+\left(v_{2} / v_{1}\right) \cdot b_{22} \cdot w_{2}\right. \\
& \left.+\left(v_{2} / v_{1}\right) \cdot b_{23} \cdot w_{3}\right) \\
b_{21 \text { new }} & =\left(v_{2} / v_{1}\right) \cdot b_{21} \\
b_{22 \text { new }} & =\left(v_{2} / v_{1}\right) \cdot b_{22} \\
b_{23 \text { new }} & =\left(v_{2} / v_{1}\right) \cdot b_{23}
\end{aligned}
$$

Therefore, WHDS after the reallocation of P1 and P2 are $b_{11 \text { new }} \sim b_{23 \text { new }}$.

\subsection{Result of calculation}

At first, the candidates of personnel reallocation are selected (ID no. 101, 102, 103 and 104 in Table1). Each of four members has different total value of skills. Moreover, the total number of permutation is 4 ! $(=24)$. The maximum values of skills are given by four cases (See Table 2,3), i.e. the result of calculation gives the same value of skills to four cases. Case 1, 2, 3, 4 are selected as the solution, and the value of skills increases from 480.00 to 504.00 . 
Table2 All pairs of personnel reallocation (24 pairs)

\begin{tabular}{|c|c|c|c|c|c|c|c|c|c|}
\hline & \multicolumn{4}{|c|}{ Pairs after alternation } & $Q_{101 \text { new }}$ & \multirow{2}{*}{$\begin{array}{r}Q_{102 \text { new }} \\
132,00\end{array}$} & \multirow{2}{*}{$\begin{array}{r}Q_{103 \text { new }} \\
116,00\end{array}$} & \multirow{2}{*}{$\begin{array}{r}Q_{104 \text { new }} \\
120,00\end{array}$} & \multirow{2}{*}{$\frac{Q_{\text {total }}}{480,00}$} \\
\hline & 101 & 102 & 103 & 104 & 112,00 & & & & \\
\hline & 101 & 102 & 104 & 103 & 112,00 & 132,00 & 120,00 & 120,00 & 484,00 \\
\hline & 101 & 103 & 102 & 104 & 112,00 & 132,00 & 132,00 & 120,00 & 496,00 \\
\hline & 101 & 103 & 104 & 102 & 112,00 & 132,00 & 120,00 & 132,00 & 496,00 \\
\hline & 101 & 104 & 102 & 103 & 112,00 & 132,00 & 132,00 & 120,00 & 496,00 \\
\hline & 101 & 104 & 103 & 102 & 112,00 & 132,00 & 116,00 & 132,00 & 492,00 \\
\hline & 102 & 101 & 103 & 104 & 132,00 & 132,00 & 116,00 & 120,00 & 500,00 \\
\hline \multirow[t]{2}{*}{ Case2 } & 102 & 101 & 104 & 103 & 132,00 & 132,00 & 120,00 & 120,00 & 504,00 \\
\hline & 102 & 103 & 101 & 104 & 132,00 & 132,00 & 116,00 & 120,00 & 500,00 \\
\hline \multirow[t]{9}{*}{ Case3 } & 102 & 103 & 104 & 101 & 132,00 & 132,00 & 120,00 & 120,00 & 504,00 \\
\hline & 102 & 104 & 101 & 103 & 132,00 & 132,00 & 116,00 & 120,00 & 500,00 \\
\hline & 102 & 104 & 103 & 101 & 132,00 & 132,00 & 116,00 & 120,00 & 500,00 \\
\hline & 103 & 101 & 102 & 104 & 116,00 & 132,00 & 132,00 & 120,00 & 500,00 \\
\hline & 103 & 101 & 104 & 102 & 116,00 & 132,00 & 120,00 & 132,00 & 500,00 \\
\hline & 103 & 102 & 101 & 104 & 116,00 & 132,00 & 116,00 & 120,00 & 484,00 \\
\hline & 103 & 102 & 104 & 101 & 116,00 & 132,00 & 120,00 & 120,00 & 488,00 \\
\hline & 103 & 104 & 101 & 102 & 116,00 & 132,00 & 116,00 & 132,00 & 496,00 \\
\hline & 103 & 104 & 102 & 101 & 116,00 & 132,00 & 132,00 & 120,00 & 500,00 \\
\hline \multirow[t]{5}{*}{ Case4 } & 104 & 101 & 102 & 103 & 120,00 & 132,00 & 132,00 & 120,00 & 504,00 \\
\hline & 104 & 101 & 103 & 102 & 120,00 & 132,00 & 116,00 & 132,00 & 500,00 \\
\hline & 104 & 102 & 101 & 103 & 120,00 & 132,00 & 116,00 & 120,00 & 488,00 \\
\hline & 104 & 102 & 103 & 102 & 120,00 & 132,00 & 116,00 & 132,00 & 500,00 \\
\hline & 104 & 103 & 101 & 102 & 120,00 & 132,00 & 116,00 & 132,00 & 500,00 \\
\hline Case1 & 104 & 103 & 102 & 101 & 120,00 & 132,00 & 132,00 & 120,00 & 504,00 \\
\hline
\end{tabular}

Table3 Optimal pairs of new comers

\begin{tabular}{|c|c|c|c|c|c|c|c|}
\hline Case & G. no. & ID no ${ }_{\text {old new }}$ & $b_{1}$ & $b_{2}$ & $b_{3}$ & $b_{\text {total }}$ & $Q$ \\
\hline & 1 & 101101 & 95 & 40 & 5 & 140 & 112,00 \\
\hline & 2 & 102102 & 100 & 50 & 15 & 165 & 132,00 \\
\hline & & 103103 & 90 & 50 & 5 & 145 & 116,00 \\
\hline & 3 & 104104 & 80 & 60 & 10 & 150 & 120,00 \\
\hline & Total & & & & & 600 & 480,00 \\
\hline \multirow{5}{*}{1} & 1 & 101104 & 102 & 43 & 5 & 150 & 120,00 \\
\hline & 2 & 102103 & 100 & 50 & 15 & 165 & 132,00 \\
\hline & & 103102 & 102 & 57 & 6 & 165 & 132,00 \\
\hline & 3 & 104101 & 80 & 60 & 10 & 150 & 120,00 \\
\hline & Total & & & & & 630 & 504,00 \\
\hline \multirow{5}{*}{2} & 1 & 101102 & 112 & 47 & 6 & 165 & 132,00 \\
\hline & 2 & 102101 & 100 & 50 & 15 & 165 & 132,00 \\
\hline & & 103104 & 93 & 52 & 5 & 150 & 120,00 \\
\hline & 3 & 104103 & 80 & 60 & 10 & 150 & 120,00 \\
\hline & Total & & & & & 630 & 504,00 \\
\hline \multirow{5}{*}{3} & 1 & 101102 & 112 & 47 & 6 & 165 & 132,00 \\
\hline & 2 & 102103 & 100 & 50 & 15 & 165 & 132,00 \\
\hline & & 103104 & 93 & 52 & 5 & 150 & 120,00 \\
\hline & 3 & 104101 & 80 & 60 & 10 & 150 & 120,00 \\
\hline & Total & & & & & 630 & 504,00 \\
\hline \multirow{5}{*}{4} & 1 & 101104 & 102 & 43 & 5 & 150 & 120,00 \\
\hline & 2 & 102101 & 100 & 50 & 15 & 165 & 132,00 \\
\hline & & 103102 & 102 & 57 & 6 & 165 & 132,00 \\
\hline & 3 & 104103 & 80 & 60 & 10 & 150 & 120,00 \\
\hline & Total & & & & & 630 & 504,00 \\
\hline
\end{tabular}

\subsection{Minimization of WHDS in the production process}

As mentioned in Section 3.1, the improvement of value of skills, caused by the optimal personnel reallocation, is expected between different production processes. Moreover, it is possible to minimize WHDS of each production process by the adjustment of the job assignment in a group ${ }^{(4)}$. In this paper, the number of daily routines is one, in order to keep the problem simple. Here, $\boldsymbol{B}^{(\mathrm{i})}(\mathrm{i}=1,2,3)$ is WHDS for each production staff, $\boldsymbol{W}^{(\mathrm{i})}(\mathrm{i}=1,2,3)$ is skill's suitability relative to the position of staff member, $\boldsymbol{V}$ is the level of skills and knowledge of production staffs, $\boldsymbol{Y}^{(\mathrm{i})}(\mathrm{i}=1,2,3)$ is the optimal WHDS, $Q$ is the value of skills based on real measured data and $Q$ ' is the optimal value of skills. As to vector $\boldsymbol{W}$ and $\boldsymbol{V}$, see Appendix.

$$
\begin{aligned}
& Q=\left(\boldsymbol{B}^{(1)} \cdot \boldsymbol{W}^{(I)} \quad \boldsymbol{B}^{(2)} \cdot \boldsymbol{W}^{(2)} \quad \boldsymbol{B}^{(3)} \cdot \boldsymbol{W}^{(3)} \quad \boldsymbol{B}^{(4)} \cdot \boldsymbol{W}^{(3)}\right) \cdot \boldsymbol{V} \\
& Q^{\prime}=\left(\boldsymbol{Y}^{(1)} \cdot \boldsymbol{W}^{(1)} \quad \boldsymbol{Y}^{(2)} \cdot \boldsymbol{W}^{(2)} \quad \boldsymbol{Y}^{(3)} \cdot \boldsymbol{W}^{(3)} \quad \boldsymbol{Y}^{(4)} \cdot \boldsymbol{W}^{(3)}\right) \cdot \boldsymbol{V}
\end{aligned}
$$

Using Eq. (18), (19), the optimal solution for each group is found. The constraints are as follows.

(a) The total value of skills of each staff should not decrease.

(b) WHDS of each production staff is under the upper limit value.

(c) The total value of WHDS for each skill in the group is under the upper limit value (i.e. the total value of expected WHDS). If there is no solution, this constraint is relaxed.

(d) Each WHDS of the candidate production staff is not over the expected WHDS in Table 3 after the personnel change.

(e) WHDS is integer, and stays between the maximum and minimum value of expected WHDS in Table 3, after the personnel change.

The objective function is the total of WHDS in a group, and the solution is WHDS of each production staff that gives the minimum value to the objective function.

The expected WHDS of Case1, Case2, Case 3 and Case 4 are given in Table 4. In the next phase, using the data in Table 4 (the left side of Table 4) as the input, the optimal solution subject to the constraints (a) to (e) (the right side of Table 4) will be searched. 
Table4 Optimal solution 
Case 1 Operation Memory Communication Total of $\mathrm{I}$ Value of skills Optimized value

\begin{tabular}{|c|c|c|c|c|c|c|c|c|c|c|c|c|c|}
\hline G. no. & ID no. & $\mathrm{i}$ & $v$ & $b^{\mathrm{i}}{ }_{1}$ & $b^{\mathrm{i}}{ }_{2}$ & $b^{\mathrm{i}}{ }_{3}$ & $b^{\mathrm{i}}{ }_{\text {total }}$ & $Q^{\mathrm{i}}$ & $y^{\mathrm{i}}{ }_{1}$ & $y^{i_{2}}$ & $y \mathrm{i}_{3}$ & $y^{\mathrm{i}}{ }_{\text {total }}$ & $Q^{\prime \text { i }}$ \\
\hline \multirow{5}{*}{1} & 1 & 1 & 1.2 & 91 & 41 & 26 & 158 & 166,34 & 90 & 36 & 32 & 158 & 172,53 \\
\hline & 11 & 2 & 1.0 & 118 & 47 & 10 & 175 & 151,43 & 118 & 52 & 5 & 175 & 149,16 \\
\hline & 104 & 3 & 0.8 & 102 & 43 & 5 & 150 & 120,00 & 102 & 43 & 5 & 150 & 120,00 \\
\hline & \multicolumn{3}{|c|}{$b^{\mathrm{i}}\left(y^{\mathrm{i}}\right)_{\mathrm{k} \text { total }:(\mathrm{k}=1,2,3)}$} & 311 & 131 & 41 & & & 310 & 131 & 42 & & \\
\hline & \multicolumn{3}{|c|}{$b(\mathrm{y})_{\text {total }}, Q\left(Q^{\prime}\right)_{\text {total }}$} & & & & 483 & 437,77 & & & & 483 & 441,69 \\
\hline \multirow{6}{*}{2} & 2 & 1 & 1.2 & 98 & 45 & 30 & 173 & 184,39 & 80 & 50 & 43 & 173 & 205,55 \\
\hline & 12 & 2 & 1.0 & 115 & 50 & 10 & 175 & 151,74 & 115 & 54 & 6 & 175 & 149,93 \\
\hline & 103 & 3 & 0.8 & 100 & 50 & 15 & 165 & 132,00 & 100 & 50 & 6 & 156 & 124,80 \\
\hline & 102 & 4 & 0.8 & 102 & 57 & 6 & 165 & 132,00 & 102 & 48 & 6 & 156 & 124,80 \\
\hline & \multicolumn{3}{|c|}{$b^{\mathrm{i}}\left(y^{\mathrm{i}}\right)_{\mathrm{k} \text { total }:(\mathrm{k}=1,2,3)}$} & 415 & 202 & 61 & & & 397 & 202 & 61 & & \\
\hline & \multicolumn{3}{|c|}{$b(\mathrm{y})_{\text {total }}, Q\left(Q^{\prime}\right)_{\text {total }}$} & & & & 678 & 600,13 & & & & 660 & 605,08 \\
\hline \multirow{5}{*}{3} & 3 & 1 & 1.2 & 100 & 50 & 25 & 175 & 181,02 & 90 & 45 & 40 & 175 & 200,16 \\
\hline & 13 & 2 & 1.0 & 110 & 45 & 25 & 180 & 163,62 & 110 & 60 & 10 & 180 & 156,82 \\
\hline & 101 & 3 & 0.8 & 80 & 60 & 10 & 150 & 120,00 & 80 & 50 & 10 & 140 & 112,00 \\
\hline & \multicolumn{3}{|c|}{$b^{\mathrm{i}}\left(y^{\mathrm{i}}\right)_{\mathrm{k}}$ total $:(\mathrm{k}=1,2,3)$} & 290 & 155 & 60 & & & 280 & 155 & 60 & 495 & 468,98 \\
\hline & \multicolumn{3}{|c|}{$b(\mathrm{y})_{\text {total }}, Q\left(Q^{\prime}\right)_{\text {total }}$} & & & & 505 & 464,64 & & & & 495 & 468,98 \\
\hline
\end{tabular}

Case 2 operation Memory Communication Total of I Value of skills Optimized value

\begin{tabular}{|c|c|c|c|c|c|c|c|c|c|c|c|c|c|}
\hline G. no. & ID no. & $\mathrm{i}$ & $v$ & $b^{\mathrm{i}}{ }_{1}$ & $b^{\mathrm{i}}{ }_{2}$ & $b^{\mathrm{i}}{ }_{3}$ & $b^{\mathrm{i}}{ }_{\text {total }}$ & $Q^{\mathrm{i}}$ & $y^{\mathrm{i}}{ }_{1}$ & $y^{\mathrm{i}}{ }_{2}$ & $y \mathrm{i}_{3}$ & $y^{\mathrm{i}}{ }_{\text {total }}$ & $Q^{\prime}{ }^{\mathrm{i}}$ \\
\hline
\end{tabular}

\begin{tabular}{|c|c|c|c|c|c|c|c|c|c|c|c|c|c|}
\hline & 4. & & & & & & total & & & $y^{i}{ }_{2}$ & $\begin{array}{l}y_{13} \\
\end{array}$ & $y^{i}{ }_{\text {total }}$ & $Q^{\prime \mathrm{i}}$ \\
\hline \multirow{5}{*}{1} & 1 & 1 & 1.2 & 91 & 41 & 26 & 158 & 166,34 & 86 & 40 & 32 & 158 & 174,49 \\
\hline & 11 & 2 & 1.0 & 118 & 47 & 10 & 175 & 151,43 & 118 & 51 & 6 & 175 & 149,62 \\
\hline & 102 & 3 & 0.8 & 112 & 47 & 6 & 165 & 132,00 & 112 & 44 & 6 & 162 & 129,60 \\
\hline & \multicolumn{3}{|c|}{$b^{\mathrm{i}}\left(y^{\mathrm{i}}\right)_{\mathrm{k} \text { total } ;(\mathrm{k}=1,2,3)}$} & 321 & 135 & 42 & 498 & & & 135 & 44 & & \\
\hline & \multicolumn{3}{|c|}{$b(\mathrm{y})_{\text {total }}, Q\left(Q^{\prime}\right)_{\text {total }}$} & & & & & 449,77 & 316 & & & 495 & 453,71 \\
\hline \multirow{7}{*}{2} & 2 & 1 & 1.2 & 98 & 45 & 30 & 173 & 184,39 & 76 & 52 & 45 & 173 & 209,41 \\
\hline & 12 & 2 & 1.0 & 115 & 50 & 10 & 175 & 151,74 & 115 & 55 & 5 & 175 & 149,48 \\
\hline & 101 & 3 & 0.8 & 100 & 50 & 15 & 165 & 132,00 & 100 & 38 & 5 & 143 & 114,40 \\
\hline & 104 & 4 & 0.8 & 93 & 52 & 5 & 150 & 120,00 & 93 & 52 & 5 & 150 & 120,00 \\
\hline & \multicolumn{3}{|c|}{$b^{\mathrm{i}}\left(y^{\mathrm{i}}\right)_{\mathrm{k} \text { total }:(\mathrm{k}=1,2,3)}$} & 406 & 197 & 60 & & & 384 & 197 & 60 & & \\
\hline & \multicolumn{3}{|c|}{$b(\mathrm{y})_{\text {total }}, Q\left(Q^{\prime}\right)_{\text {total }}$} & & & & 663 & 588,13 & & & & 641 & 593,29 \\
\hline & 3 & 1 & 1.2 & 100 & 50 & 25 & 175 & 181,02 & 90 & 45 & 40 & 175 & 200,16 \\
\hline \multirow[t]{4}{*}{3} & 13 & 2 & 1.0 & 110 & 45 & 25 & 180 & 0,00 & 110 & 60 & 10 & 180 & 156,82 \\
\hline & 103 & 3 & 0.8 & 80 & 60 & 10 & 150 & 120,00 & 80 & 50 & 10 & 140 & 112,00 \\
\hline & \multicolumn{3}{|c|}{$b^{\mathrm{i}}\left(y^{\mathrm{i}}\right)_{\mathrm{k} \text { total }:(\mathrm{k}=1,2,3)}$} & 290 & 155 & 60 & & & 280 & 155 & 60 & & \\
\hline & \multicolumn{3}{|c|}{$b(\mathrm{y})_{\text {total }}, Q\left(Q^{\prime}\right)_{\text {total }}$} & & & & 505 & 464,64 & & & & 495 & 468,98 \\
\hline & & & & & & & & Total & HDS, tot & alue of & kills & \begin{tabular}{|l|l|}
1631 \\
\end{tabular} & 1515,98 \\
\hline
\end{tabular}

Case 3

of skills Optimized value

\begin{tabular}{|c|c|c|c|c|c|c|c|c|c|c|c|c|c|}
\hline G. no. & ID no. & $\mathrm{i}$ & $v$ & $b^{\mathrm{i}}{ }_{1}$ & $b_{2}^{\mathrm{i}}$ & $b_{3}^{\mathrm{i}}$ & $b^{\mathrm{i}}$ total & $Q^{\mathrm{i}}$ & $y^{\text {i }}{ }_{1}$ & $y_{2}^{\mathrm{i}}$ & $y \mathbf{i}_{3}$ & $y^{\mathrm{i}}{ }_{\text {total }}$ & $Q^{\prime}{ }^{\mathrm{i}}$ \\
\hline \multirow{5}{*}{1} & 1 & 1 & 1.2 & 91 & 41 & 26 & 158 & 166,34 & 86 & 40 & 32 & 158 & 174,49 \\
\hline & 11 & 2 & 1.0 & 118 & 47 & 10 & 175 & 151,43 & 118 & 51 & 6 & 175 & 149,62 \\
\hline & 102 & 3 & 0.8 & 112 & 47 & 6 & 165 & 132,00 & 112 & 44 & 6 & 162 & 129,60 \\
\hline & \multicolumn{3}{|c|}{$b^{\mathrm{i}}\left(y^{\mathrm{i}}\right)_{\mathrm{k} \text { total }:(\mathrm{k}=1,2,3)}$} & 321 & 135 & 42 & & & 316 & 135 & 44 & & \\
\hline & \multicolumn{3}{|c|}{$b(\mathrm{y})_{\text {total }}, Q\left(Q^{\prime}\right)_{\text {total }}$} & & & & 498 & 449,77 & & & & 495 & 453,71 \\
\hline \multirow{7}{*}{2} & 2 & 1 & 1.2 & 98 & 45 & 30 & 173 & 184,39 & 76 & 52 & 45 & 173 & 209,41 \\
\hline & 12 & 2 & 1.0 & 115 & 50 & 10 & 175 & 151,74 & 115 & 55 & 5 & 175 & 149,48 \\
\hline & 103 & 3 & 0.8 & 100 & 50 & 15 & 165 & 132,00 & 100 & 38 & 5 & 155 & 124,00 \\
\hline & 104 & 4 & 0.8 & 93 & 52 & 5 & 150 & 120,00 & 93 & 52 & 5 & 137 & 109,60 \\
\hline & \multicolumn{3}{|c|}{$b^{\mathrm{i}}\left(y^{\mathrm{i}}\right)_{\mathrm{k} \text { total }:(\mathrm{k}=1,2,3)}$} & 406 & 197 & 60 & & & 384 & 197 & 60 & & \\
\hline & \multicolumn{3}{|c|}{$b(\mathrm{y})_{\text {total }}, Q\left(Q^{\prime}\right)_{\text {total }}$} & & & & 663 & 588,13 & & & & 641 & 593,29 \\
\hline & 3 & 1 & 1.2 & 100 & 50 & 25 & 175 & 181,02 & 90 & 45 & 40 & 175 & 200,16 \\
\hline \multirow[t]{4}{*}{3} & 13 & 2 & 1.0 & 110 & 45 & 25 & 180 & 163,62 & 110 & 60 & 10 & 180 & 156,82 \\
\hline & 101 & 3 & 0.8 & 80 & 60 & 10 & 150 & 120,00 & 80 & 50 & 10 & 140 & 112,00 \\
\hline & \multicolumn{3}{|c|}{$b^{\mathrm{i}}\left(y^{\mathrm{i}}\right)_{\mathrm{k} \text { total }:(\mathrm{k}=1,2,3)}$} & 290 & 155 & 60 & & & 280 & 155 & 60 & & \\
\hline & \multicolumn{3}{|c|}{$b(\mathrm{y})_{\text {total }}, Q\left(Q^{\prime}\right)_{\text {total }}$} & & & & 505 & 464,64 & & & & 495 & 468,98 \\
\hline & & & & & & & & \multicolumn{4}{|c|}{ Total WHDS, total value of skills } & 1631 & 1515,98 \\
\hline
\end{tabular}

Case 4

Total WHDS, total value of skills

\begin{tabular}{|c|c|c|c|c|c|c|c|c|c|c|c|c|c|}
\hline G. no. & ID no. & $\mathrm{i}$ & $v$ & $b^{\mathrm{i}}{ }_{1}$ & $b^{\mathrm{i}}{ }_{2}$ & $b^{\mathrm{i}}{ }_{3}$ & $b^{\mathrm{i}}{ }_{\text {total }}$ & $Q^{\mathrm{i}}$ & $y^{i}{ }_{1}$ & $y^{\mathrm{i}}{ }_{2}$ & $y \mathrm{i}_{3}$ & $y^{\mathrm{i}}{ }_{\text {total }}$ & $Q^{\prime}{ }^{\mathrm{i}}$ \\
\hline & 1 & 1 & 1.2 & 91 & 41 & 26 & 158 & 166,34 & 90 & 36 & 32 & 158 & 172,53 \\
\hline 1 & 11 & 2 & 1.0 & 118 & 47 & 10 & 175 & 151,43 & 118 & 52 & 5 & 175 & 149,16 \\
\hline & 104 & 3 & 0.8 & 102 & 43 & 5 & 150 & 120,00 & 102 & 43 & 5 & 150 & 120,00 \\
\hline & $b^{\mathrm{i}}\left(y^{\mathrm{i}}\right)_{\mathrm{k}}$ & otal : & $=1,2,3)$ & 311 & 131 & 41 & & & 310 & 131 & 42 & & \\
\hline & $b(\mathrm{y})_{\text {tota }}$ & $Q$ & 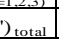 & & & & 483 & 437,77 & & & & \begin{tabular}{|l|}
483 \\
\end{tabular} & 441,69 \\
\hline & 2 & 1 & 1.2 & 98 & 45 & 30 & 173 & 184,39 & 80 & 50 & 43 & \begin{tabular}{|l|}
173 \\
\end{tabular} & 205,55 \\
\hline 2 & 12 & 2 & 1.0 & 115 & 50 & 10 & 175 & 151,74 & 115 & 54 & 6 & 175 & 149,93 \\
\hline & 101 & 3 & 0.8 & 100 & 50 & 15 & 165 & 132,00 & 100 & 50 & 6 & 156 & 124,80 \\
\hline & 102 & 4 & 0.8 & 102 & 57 & 6 & 165 & 132,00 & 102 & 48 & 6 & 156 & 124,80 \\
\hline & $b^{\mathrm{i}}\left(y^{\mathrm{i}}\right)_{\mathrm{k}}$ & otal : & $=1,2,3)$ & 415 & 202 & 61 & & & 397 & 202 & 61 & & \\
\hline & $b(\mathrm{y})_{\text {tota }}$ & $Q$ & ') $)_{\text {total }}$ & & & & 678 & 600,13 & & & & 660 & 605,08 \\
\hline & 3 & 1 & 1.2 & 100 & 50 & 25 & 175 & 181,02 & 90 & 45 & 40 & 175 & 200,16 \\
\hline 3 & 13 & 2 & 1.0 & 110 & 45 & 25 & 180 & 163,62 & 110 & 60 & 10 & 180 & 156,82 \\
\hline & 103 & 3 & 0.8 & 80 & 60 & 10 & 150 & 120,00 & 80 & 50 & 10 & 140 & 112,00 \\
\hline & $b^{\mathrm{i}}\left(y^{\mathrm{i}}\right)_{\mathrm{k}}$ & tal : & $=1,2.3)$ & 290 & 155 & 60 & & & 280 & 155 & 60 & \begin{tabular}{l|}
495 \\
\end{tabular} & 468,98 \\
\hline & $b(\mathrm{y})_{\text {tota }}$ & $Q$ & & & & & 505 & 464,64 & & & & 495 & 468,98 \\
\hline & & & & & & & & Tot & HDS & 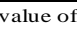 & & \begin{tabular}{|l|l|}
1638 \\
\end{tabular} & 1515,75 \\
\hline
\end{tabular}

Candidates of allocation change

Maximum of expected WHDS: $b^{\mathrm{i}}{ }_{\mathrm{k} \text { max }}$

Table 5 Total WHDS and value of skills for each case. 


\begin{tabular}{c|cc}
\hline Case & $\boldsymbol{B}_{\boldsymbol{C} \text { ase_total }}$ & $\boldsymbol{Q}_{\text {Case } \text { total }}$ \\
\hline & 1636 & 1478,54 \\
\hline 1 & 1638 & 1515,75 \\
\hline 2 & 1631 & 1515,98 \\
\hline 3 & 1631 & 1515,98 \\
\hline 4 & 1638 & 1515,75 \\
\hline
\end{tabular}

As the final solution, Case 2, 3 give the minimum WHDS and the maximum value of skills. Table 5 shows the total WHDS and value of skills of each case based on the results in Table 4.

\section{Conclusion}

The production staffs that practice the daily routine in the production process make efforts to improve the skills and knowledge, in order to maintain and to increase the productivity. In addition to the education or the training in a group, it is effective to work for several production processes to improve the skills and knowledge ${ }^{(5,6)}$. Therefore, the personnel reallocation is often done in various production processes. However, the way that the production process or the production staff is selected for the personnel reallocation does not have a clear rule. The reason why, typically, the selection is not quantitative is that the quantitative estimation of skills is difficult. Nevertheless, it is possible to estimate the skills quantitatively ${ }^{(3)}$, and according to this metric the adjustment of the job routine assignment in the production process permits the reduction of WHDS without a decrease of the total value of skills ${ }^{(4)}$. In this paper, by expanding the object of estimation of the value of skills to different production processes, it is shown that the personnel reallocation causes an increase of the value of skills with the reduction of WHDS. Moreover, the increase of skills and knowledge of production staff permits to improve the operational resilience of the production process $(5,6)$. Especially, in the managerial subjects of organization (Individual, Group and Company), the group is important for the improvement of the operational resilience ${ }^{(7)}$. The possibility to produce the margin of WHDS, and consequently generate the margin of work hours, used to improve the skills and knowledge of production staff through the personnel reallocation, is confirmed by the example. The next step of this research is to analyze the daily routine more widely and deeply, to measure the value of skills after the personnel reallocation and to verify the validity of the personnel reallocation.

\section{Acknowledgements}

This research was partially supported by the Ministry of Education, Science, Sports and Culture, Grant-in-Aid for Scientific Research (B), No.24310119 (2012) and (B), No.25282101 (2013).

\section{References}

(1) M. Noda, K. Kuratsune, and H. Nishitani, "Quantitative evaluation of ability of shift of multi-skilled operators to cope with abnormal plant conditions", Human Factors in Japan, Vol.12, No.2, pp. 102-109, 2008

(2) M. Noda and K. Kuratsune, and H. Nishitani, "Optimal assignment of plant operators on basis of shift's ability evaluation", Human Factors in Japan, Vol.14, No.2, pp. 72-78, 2010

(3) H. Eguchi and D. O'Donovan, "Advancement and Transfer of skills by Reciprocal Interaction of Human Skills and Technique via a Production Support System in the Chemical Industry", J. Chem. Eng. Japan, Vol.43, pp. 435-442, 2010

(4) H. Eguchi, T. Aoyama, K. Seki, I. Koshijima, “Optimal job routine assignment for the improvement of operational resilience based on skills and knowledge of production staff in the chemical industry" (Unpublished)

(5) H. Eguchi, T. Aoyama, K. Seki, D. O’Donovan, I. Koshijima, "Impact of Organizational Structure on Resilience of Production Process based on human factors in the Chemical Industry," SOMChE \& RSCE 2014 Vol.1, PSE053, 2014

(6) H. Eguchi, T. Aoyama, K. Seki, D. O’Donovan, I. Koshijima, "A Metric for quantitative estimation of Production process resilience based on the skills and knowledge of Production plant personnel in the Chemical industry" (Unpublished)

(7) F. Bracco, T. Piccinno and G. Dorigatti, "Turning Variability into Emergent Safety: the Resilience Matrix for Providing Strong Responses to Weak Signals", Proceedings of $5^{\text {TH }}$ SYMPOSIUM ON RESILIENCE ENGINEERING MANAGING TRADE-OFF, pp. 23-28, 2013

(8) H. Eguchi, and D. O'Donovan, "Evaluation of labor productivity using a corrected production staff number approach in the chemical industry", J. Chem. Eng. Japan, Vol. 45, pp. 206-209, 2012

\section{Appendix: Formalization and optimization}


The WHDS of three production staffs in group 1 is vector $\boldsymbol{B}^{(\mathrm{i})}$ ( $i=1$ supervisor, $i=2$ deputy-supervisor, $i=3,4$ new comer). The weighting vector based on skill's suitability relative to the position of the staff member is vector $\boldsymbol{W}^{(\mathrm{i})}(\mathrm{i}=1,2,3)^{(8)}$. The level of skills and knowledge of production staff is vector $\boldsymbol{V}^{\left({ }^{(8)}\right.}$. The WHDS for which the summation of component is minimum is matrix $\boldsymbol{Y}^{(\mathrm{i})}(\mathrm{i}=1,2,3,4)$. Moreover, $Q$ is the Value of skills based on real measured data and $Q$ ' is the optimal value of skills.

$$
\begin{aligned}
& \boldsymbol{B}^{(\mathrm{i})}=\left(\begin{array}{lll}
b_{1}^{\mathrm{i}} & b_{2}^{\mathrm{i}} & b_{3}^{\mathrm{i}}
\end{array}\right) \quad(\mathrm{i}=1,2,3,4) \\
& \boldsymbol{W}^{(1)}=\left(\begin{array}{lll}
0.5740 & 0.9823 & 1.7735
\end{array}\right)^{T} \\
& W^{(2)}=\left(\begin{array}{lll}
0.8054 & 0.9098 & 1.3633
\end{array}\right)^{T} \\
& \left.\boldsymbol{W}^{(3)}=\left(\begin{array}{lll}
1.0 & 1.0 & 1.0
\end{array}\right)\right)^{T} \\
& \boldsymbol{V}=\left(\begin{array}{lll}
1.2 & 1.0 & 0.8
\end{array}\right)^{T} \\
& Q=\left(\boldsymbol{B}^{(1)} \cdot \boldsymbol{W}^{(1)} \quad \boldsymbol{B}^{(2)} \cdot \boldsymbol{W}^{(2)} \quad \boldsymbol{B}^{(3)} \cdot \boldsymbol{W}^{(3)} \quad \boldsymbol{B}^{(4)} \cdot \boldsymbol{W}^{(3)}\right) \cdot \boldsymbol{V} \\
& =Q_{\text {total }} \\
& \boldsymbol{Y}^{(\mathrm{i})}=\left(\begin{array}{lll}
y_{1}^{i} & y_{2}^{i} & y_{3}^{i}
\end{array}\right) \quad(\mathrm{i}=1,2,3,4)
\end{aligned}
$$

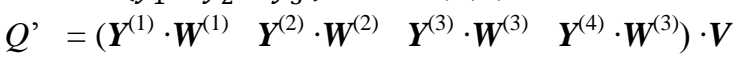

$$
\begin{aligned}
& =0.6888 y_{1}^{1}+1.0828 y_{2}^{1}+2.1282 y_{3}^{1}+0.8054 y_{1}^{2}+ \\
& 0.9098 y_{2}^{2}+1.3633 y_{3}^{2}+0.8 y_{1}^{3}+0.8 y_{2}^{3}+ \\
& 0.8 y_{3}^{3}+0.8 y_{1}^{4}+0.8 y_{2}^{4}+0.8 y_{3}^{4}
\end{aligned}
$$

\section{Constraints}

1) The total value of skills of each staff should not decrease. $Q^{\prime} \geq Q_{\text {total }}$

2) WHDS of each production staff is under the upper limit value.

$$
\begin{aligned}
& \sum_{k=1}^{3} y_{k}^{1} \leq b_{\text {total }}^{1} \\
& \sum_{k=1}^{3} y_{k}^{2} \leq b_{\text {total }}^{2}
\end{aligned}
$$

$$
\begin{aligned}
& \sum_{k=1}^{3} y_{k}^{3} \leq b_{\text {total }}^{3} \\
& \sum_{k=1}^{3} y_{k}^{4} \leq b_{\text {total }}^{4}
\end{aligned}
$$

3) The total value of WHDS for each skill in a group is under the upper limit value (i.e. the total value of expected WHDS). If there is no solution, this constraint is relaxed.

$\sum_{i=1}^{3} y_{1}^{i} \leq b_{1 \text { total }}$

$\sum_{i=1}^{3} y_{2}^{i} \leq b_{2 \text { total }}$

$\sum_{i=1}^{3} y_{3}^{i} \leq b_{3 \text { total }}$

4) Each WHDS of the candidate production staff is not over the expected WHDS after the personnel change in Table 4 . The suffix $i$ indicates the candidate. In case of Group 1 and 3, i=3; while in case of Group 2, i=3,4.

$y_{1}^{i} \leq b_{1}^{\mathrm{i}}$

$y_{2}^{i} \leq b_{2}^{\mathrm{i}}$

$y_{3}^{i} \leq b_{3}^{\mathrm{i}}$

5) WHDS $y_{k}^{i}(\mathrm{i}=1,2,3,4 ; \mathrm{k}=1,2,3)$ is integer, and stays between the maximum and minimum of the expected WHDS after the personnel change in Table 4.

$b_{\mathrm{k} \text { min }}^{\mathrm{i}} \leq y_{k}^{i} \leq b_{\mathrm{k} \max }^{\mathrm{i}}(\mathrm{i}=1,2,3,4 ; \mathrm{k}=1,2,3)$

\section{Objective function}

The objective function is the total of WHDS in a group $J$, and the solution is WHDS of each production staff $\boldsymbol{Y}^{(\mathrm{i})}$ $(i=1,2,3,4)$ that give the minimum value to the objective function.

$$
J=\sum_{i=1}^{4} \sum_{k=1}^{3} y_{k}^{i}
$$

In this study, for the solver of optimization, Solver @Microsoft inc. is used. 